\title{
SUBDIRECTLY IRREDUCIBLE DIFFERENTIAL MODES
}

\author{
DAVID STANOVSKÝ
}

\begin{abstract}
We study a variety of modes (idempotent algebras with mutually commuting term operations), so called differential modes, having a strongly solvable chain $0 \leq \alpha \leq 1$ in their congruence lattices. We show an explicit description of subdirectly irreducible algebras in this variety, and use it to compute residual bounds of its subvarieties. It follows from our results that all subvarieties with a finite residual bound are finitely based.
\end{abstract}

\section{INTRODUCTION}

Modes are idempotent algebras with a commutative clone of term operations, or, in other terms, idempotent algebras where all term operations are homomorphisms. We are interested in so called ( $n$-ary) (left) differential modes, a structuraly simple yet non-trivial subclass of strongly solvable modes, consisting of the modes that possess a congruence such that all its blocks and the factor are left projection algebras. Equivalently, this is a variety axiomatized by the following three identities:

$$
\begin{aligned}
f(x, x, \ldots, x) & =x \\
f\left(f\left(x, y_{2}, \ldots, y_{n}\right), z_{2}, \ldots, z_{n}\right) & =f\left(f\left(x, z_{2}, \ldots, z_{n}\right), y_{2}, \ldots, y_{n}\right) \\
f\left(x, f\left(y_{21}, \ldots, y_{2 n}\right), \ldots, f\left(y_{n 1}, \ldots, y_{n n}\right)\right) & =f\left(x, y_{21}, \ldots, y_{n 1}\right)
\end{aligned}
$$

The study of general differential modes was initiated in [5], which explains our motivation and contains the following results: alternative axiomatizations, a description of reducts, a decomposition based on the congruence $\lambda$ (to be defined in Section 2), a description of free algebras, including normal forms of terms, a proof that finitely based subvarieties are relatively 1-based, and an example of a locally finite subvariety with no finite base for its identities. The second paper of the series [7] contains a thorough discussion of the lattice of subvarieties of Szendrei differential modes, including the fact that all of them are finitely based (actually in two variables).

However, the study of binary differential modes started much earlier in $[9,10,12]$ (alternative names, such as LIR-groupoids, were used) and also in [8, 11], taking care of a special case, when all translations are permutations ( $n$-cyclic groupoids). The binary case is significantly simpler, because binary modes are Szendrei modes (see Section 4 for a discussion). The results of $[9,10]$ and some of [12] were appropriately generalized in $[5,7]$, however our previous two papers did not address subdirectly irreducible algebras. The present paper fills the gap. One of the main theorems of [11], the description of subdirectly irreducible binary differential modes,

2000 Mathematics Subject Classification. 03C05, 08A05, $08 \mathrm{~B} 26$.

Key words and phrases. Differential mode, subdirectly irreducible, residual bound.

The work is a part of the research project MSM 0021620839 financed by MŠMT ČR and partly supported by the grant GAČR 201/08/P056. 
where all translations are permutations of a bounded order, is a special case of our Corollary 4.2 (the cocyclic case). The ideas behind our construction of $\mathbf{B} \propto_{F} \mathbf{C}$ and Proposition 3.3 can be traced in $[8,11]$.

My personal interest in strongly solvable modes comes from the desire to understand what are (finite) modes. There are two results by Keith Kearnes addressing this question. In [2], he proved that every locally finite variety $\mathcal{V}$ of modes decomposes into three subvarieties $\mathcal{V}_{1}, \mathcal{V}_{2}$ and $\mathcal{V}_{5}$, consisting of the strongly solvable modes, the affine modes and the semilattice modes in $\mathcal{V}$. In [4], he proved that subdirectly irrecudible modes come in three very different families, corresponding to the three properties. While the latter two types are well understood [3, 13], a little is known about the strongly solvable case. Differential modes are a natural class to begin with.

The main result of the present paper is an explicit description of subdirectly irreducible differential modes (Theorem 3.5). We use it to determine which varieties of Szendrei differential modes have finite residual bound (Theorem 5.5), and prove that no non-Szendrei variety has finite residual bound (Theorem 5.4). As a consequence, we obtain that Park's conjecture holds for differential modes. The discussion of residual bounds fundamentally relies on the description of subdirectly irreducible commutative unary algebras by Z. Ésik and B. Imreh [1].

The paper is organized as follows. In Section 2, we briefly recall what we need from the previous papers on differential modes, particularly the Mal'tsev decomposition to projection algebras. Most facts are presented in a slightly novel way. Section 3 contains the description of subdirectly irreducible differential modes, and the next section its specialization to Szendrei differential modes. In Section 5, we discuss the residual bounds. The final section contains remarks on how the results relate to other problems in universal algebra.

\section{DeComposition THEOREM}

To improve clarity of the text, we will consider ternary algebras in this paper, with the (only) operation denoted simply by brackets, avoiding commas. It means,

$$
(x y z)
$$

will stand for the result of the single basic operation on arguments $x, y, z$. Our results can be generalized to other arities in an obvious way. (As noted above, the binary case does not capture full generality.)

(Ternary) modes are characterized by the following two identities: the idempotent law

$$
(x x x)=x
$$

and the entropic law

$$
\left(\left(x_{11} x_{12} x_{13}\right)\left(x_{21} x_{22} x_{23}\right)\left(x_{31} x_{32} x_{33}\right)\right)=\left(\left(x_{11} x_{21} x_{31}\right)\left(x_{12} x_{22} x_{32}\right)\left(x_{13} x_{23} x_{33}\right)\right) .
$$

A mode is called (left) differential (for a reason explained in [12]), if it also satisfies the (left) reductive law

$$
\left(x\left(y u_{1} u_{2}\right)\left(z v_{1} v_{2}\right)\right)=(x y z) .
$$

In left reductive algebras, every term is equivalent to a term in the left reduced form

$$
\left(\left(\left(\left(x y_{1} z_{1}\right) y_{2} z_{2}\right) \ldots\right) y_{n} z_{n}\right) \text {. }
$$


In particular, the entropic law is equivalent to the identity

(E)

$$
((x y z) u v)=((x u v) y z) .
$$

By a left projection algebra (or left zero algebra) we mean an algebra that satisfies the identity

$$
(x y z)=x .
$$

In the sequel, we shall omit the adjective "left". Indeed, we could have chosen any coordinate to be the distinguished one.

Let $\mathbf{A}$ be a ternary algebra and $a, b \in A$. By a (right) translation we mean the mapping

$$
R_{a b}: A \rightarrow A, \quad x \mapsto(x a b) .
$$

The translations generate the (right) multiplication monoid of $\mathbf{A}$, denoted by

$$
\mathbf{R}_{\mathbf{A}}=\left\langle R_{a b}: a, b \in A\right\rangle .
$$

In differential modes, $\mathbf{R}_{\mathbf{A}}$ is a commutative submonoid of the endomorphism monoid: indeed, (E) is equivalent to the fact that translations are mutually commutative, and $(\mathrm{E})$ and $(\mathrm{R})$ respectively give that

$$
R_{a b}((x y z))=((x y z) a b)=((x a b) y z)=((x a b)(y a b)(z a b))=\left(R_{a b}(x), R_{a b}(y), R_{a b}(z)\right) .
$$

Consequently, the images of the translations are subalgebras of $\mathbf{A}$. We define the orbit of an element $a$ by

$$
R_{\mathbf{A}}(a)=\left\{\varphi(a): \varphi \in R_{\mathbf{A}}\right\} .
$$

It also forms a subalgebra of $\mathbf{A}:\left(\varphi_{1}(a) \varphi_{2}(a) \varphi_{3}(a)\right)=\left(\varphi_{1}(a) a a\right)=\varphi_{1}((a a a))=$ $\varphi_{1}(a)$, so it is actually a left projection subalgebra. Now comes a crucial structural concept in the theory of differential modes: we define a relation $\lambda$ by

$$
a \lambda b \quad \text { iff } \quad R_{\mathbf{A}}(a) \cap R_{\mathbf{A}}(b) \neq \emptyset .
$$

In other words, $a \lambda b$ iff there are $\varphi, \psi \in R_{\mathbf{A}}$ such that $\varphi(a)=\psi(b)$.

Proposition 2.1. Let $\mathbf{A}$ be a differential mode. Then

(1) $\lambda$ is the smallest congruence such that $\mathbf{A} / \lambda$ is a projection algebra;

(2) if $b_{i} \lambda c_{i}, i=1,2$, then $\left(a b_{1} b_{2}\right)=\left(a c_{1} c_{2}\right)$. Particularly, the blocks of $\lambda$ are projection algebras.

Proof. (1) Let us first show that $\lambda$ is a congruence. Reflexivity and symmetry is obvious, and if $\varphi(a)=\psi(b)$ and $\chi(b)=v(c)$, then, by $(\mathrm{E}), \chi \varphi(a)=\chi \psi(b)=$ $\psi \chi(b)=\psi v(c)$. If $a_{i} \lambda b_{i}, i=1,2,3$, then $\left(a_{1} a_{2} a_{3}\right) \lambda a_{1} \lambda b_{1} \lambda\left(b_{1} b_{2} b_{3}\right)$, hence $\lambda$ is a congruence. Now, let $\alpha$ be the congruence generated by the pairs $(R(a), a)$, for every $a \in A$ and every translation $R$. Since $\alpha \leq \lambda$, we immediately see that $\mathbf{A} / \lambda$ is a projection algebra. Are the two congruences equal? Assume $a \lambda b$ with $\varphi(a)=\psi(b)$ for some $\varphi=R_{1} \cdots R_{n} \in R_{\mathbf{A}}$ and $\psi=R_{1}^{\prime} \cdots R_{m}^{\prime} \in R_{\mathbf{A}}$, where the $R_{i}$ and $R_{i}^{\prime}$ are translations. Then $a \alpha R_{n}(a) \alpha R_{n-1} R_{n}(a) \alpha \ldots \alpha R_{1} \cdots R_{n}(a)=$ $R_{1}^{\prime} \ldots R_{m}^{\prime}(b) \alpha \ldots \alpha R_{m}^{\prime}(b) \alpha b$. Hence $\alpha=\lambda$.

(2) Assume $\varphi_{i}\left(b_{i}\right)=\psi_{i}\left(c_{i}\right), i=1,2$. It follows from (R) that $\left(a b_{1} b_{2}\right)=$ $\left(a \varphi_{1}\left(b_{1}\right) \varphi_{2}\left(b_{2}\right)\right)=\left(a \psi_{1}\left(c_{1}\right) \psi_{2}\left(c_{2}\right)\right)=\left(a c_{1} c_{2}\right)$. For the latter claim, put $c_{1}=c_{2}=$ $a$. 
Let $\alpha \leq \beta$ be congruences of an algebra $\mathbf{A}$. We say that $\beta$ is strongly abelian over $\alpha$, if for every term $t$ and all tuples $\bar{a}, \bar{b}, \bar{c}$ such that $a_{i} \beta b_{i} \beta c_{i}$ for every $i$

$$
t\left(a_{1}, a_{2}, \ldots, a_{n}\right) \alpha t\left(b_{1}, b_{2} \ldots, b_{n}\right) \text { implies } t\left(a_{1}, c_{2}, \ldots, c_{n}\right) \alpha t\left(b_{1}, c_{2} \ldots, c_{n}\right) .
$$

An algebra is called strongly abelian, if the largest congruence 1 is strongly abelian over the smallest congruence 0 . Projection algebras are strongly abelian, since every term depends on exactly one variable. Conversely, projection algebras are the only strongly abelian differential modes. Consider $t(x, y, z)=(x y z)$ : since $t(a, b, c)=t((a b c),(a b c),(a b c))$, strong abelianess gives $t(a, a, a)=t((a b c), a, a)$, and so $a=((a b c) a a)=((a a a) b c)=(a b c)$ using $(\mathrm{E})$ and idempotency. (In general, an idempotent algebra is strongly abelian if and only if it is a direct product of projection algebras on possibly different coordinates.)

Proposition 2.2. Let $\mathbf{A}$ be a differential mode. Then $\lambda$ is strongly abelian over 0 .

Proof. Consider a term $t\left(x_{1}, x_{2}, \ldots, x_{n}\right)$ and tuples $\bar{a}, \bar{b}, \bar{c}$ such that $a_{i} \lambda b_{i} \lambda c_{i}$ for every $i$. We can assume that $t$ is in the left reduced form and we shall check two cases. First, let $x_{1}$ be the leftmost variable. Then, assuming $t\left(a_{1}, \ldots, a_{n}\right)=$ $t\left(b_{1}, \ldots, b_{n}\right)$ and using Proposition 2.1(2) recursively, we obtain $t\left(a_{1}, c_{2}, \ldots, c_{n}\right)=$ $t\left(a_{1}, a_{2}, \ldots, a_{n}\right)=t\left(b_{1}, b_{2}, \ldots, b_{n}\right)=t\left(b_{1}, c_{2}, \ldots, c_{n}\right)$. In the other case, let $x_{k}$ be the leftmost variable for some $k>1$. Then we do not even need the premise of the implication, and, again, using Proposition 2.1(2) recursively, we obtain $t\left(a_{1}, c_{2}, \ldots, c_{n}\right)=t\left(b_{1}, c_{2}, \ldots, c_{n}\right)$.

An algebra is called strongly solvable of length $n$, if there are congruences $0=$ $\alpha_{0} \leq \alpha_{1} \leq \ldots \leq \alpha_{n}=1$ such that $\alpha_{i+1}$ is strongly abelian over $\alpha_{i}$, for every $i$.

Theorem 2.3. Let $\mathbf{A}$ be a ternary mode. Then the following are equivalent:

(1) A satisfies the identity $(R)$.

(2) A has a congruence $\alpha$ such that $\mathbf{A} / \alpha$ and all blocks of $\alpha$ are projection algebras.

Moreover, if the conditions are satisfied, then $\mathbf{A}$ is strongly solvable of length 2.

Proof. (1) $\Rightarrow$ (2) follows from Proposition 2.1, put $\alpha=\lambda$.

$(2) \Rightarrow(1)$. Using idempotency and entropicity,

$$
\left(x\left(y u_{1} u_{2}\right)\left(z v_{1} v_{2}\right)\right)=\left((x x x)\left(y u_{1} u_{2}\right)\left(z v_{1} v_{2}\right)\right)=\left((x y z)\left(x u_{1} v_{1}\right)\left(x u_{2} v_{2}\right)\right) .
$$

Now $(x a b) \alpha x$ for any $a, b$, because the factor is a projection, and so

$$
\left((x y z)\left(x u_{1} v_{1}\right)\left(x u_{2} v_{2}\right)\right)=(x y z)
$$

because the blocks of $\alpha$ are projections.

Finally, note that $0 \leq \lambda \leq 1$ is a strongly solvable chain in the congruence lattice of $\mathbf{A}$ : The case $0 \leq \lambda$ is discussed in Proposition 2.2, and 1 is strongly abelian over $\lambda$, because $\mathbf{A} / \lambda$ is a projection algebra, hence strongly abelian.

Consequently, the variety of differential modes is the Mal'tsev product of the variety of projection algebras over itself, relative to modes (this means, by definition, the variety of modes satisfying the condition (2)). 


\section{DESCRIPTION OF SUBDIRECTLY IRREDUCIBLES}

We recall that an algebra $\mathbf{A}$ is subdirectly irreducible (or briefly $S I$ ), if it cannot be decomposed as a subdirect product in a non-trivial way. Equivalently, if the intersection of all non-trivial congruences is non-trivial. It means, $\mathbf{A}$ has the smallest non-trivial congruence, called the monolith of $\mathbf{A}$.

Simple algebras are indeed subdirectly irreducible. It follows from Theorem 2.3 that the only simple differential mode is the two-element projection algebra. It generates the only minimal subvariety of differential modes, the variety of projection algebras. A differential mode is called proper, if it is not a projection algebra.

We start with a construction of differential modes. Let $B, C$ be disjoint nonempty sets, and $g_{c}, h_{c}, f_{c d}: B \rightarrow B$ mappings, for every $c, d \in C$. Put

$$
A=B \cup C
$$

and define a ternary operation (_. - ) by

$$
(c u v)=c, \quad\left(b b_{1} b_{2}\right)=b, \quad\left(b b_{1} c\right)=g_{c}(b), \quad\left(b c b_{1}\right)=h_{c}(b), \quad(b c d)=f_{c d}(b)
$$

for every $b, b_{1}, b_{2} \in B, c, d \in C$ and $u, v \in A$. The algebra $\left(A,\left({ }_{--}\right)\right)$will be denoted

$$
\mathbf{B} \propto_{F} \mathbf{C},
$$

where $F$ stands for the collection $\left(g_{c}, h_{c}, f_{c d}: c, d \in C\right)$ of the defining mappings. When we write $\mathbf{B} \propto_{F} \mathbf{C}$ for some $F$, we implicitly assume that $F$ is admissible for the construction, and we shall use the above notation for the mappings.

Lemma 3.1. The algebra $\mathbf{B} \propto_{F} \mathbf{C}$ is a differential mode if and only if the mappings from $F$ are mutually commutative.

Proof. The identity $(\mathrm{R})$ follows from the fact that right translations on the elements of $C$ act as the identity, and right translations on the elements of $B$ do not depend on the parameters from $B$. The identity $(\mathrm{E})$ is equivalent to the fact that the mappings are mutually commutative.

We prove that every SI differential mode can be constructed in this way.

Lemma 3.2. In every SI differential mode, $\lambda$ has exactly one non-trivial block (i.e., with more than 1 element).

Proof. Consider two non-trivial blocks $U, V$ of $\lambda$ and put $\rho=U^{2} \cup i d$ and $\sigma=V^{2} \cup i d$. Since $\rho, \sigma \subseteq \lambda$, Proposition 2.1(2) shows that both are congruences of $\mathbf{A}$. They are non-trivial, but have a trivial intersection, hence the algebra is not SI.

Proposition 3.3. Let $\mathbf{A}$ be a proper SI differential mode. Then $\mathbf{A}=\mathbf{B} \propto_{F} \mathbf{C}$, where $B$ is the unique non-trivial block of $\lambda, C=A \backslash B$, and $g_{c}, h_{c}, f_{c d}: B \rightarrow B$ are defined by $g_{c}(b)=(b b c), h_{c}(b)=(b c b)$ and $f_{c d}(b)=(b c d)$.

Proof. Since $(c u v) \lambda c$ for all $c \in C, u, v \in A$, we see that $(c u v)=c$. And it follows from Proposition 2.1(2) that, on elements of $B$, the translations do not depend on their parameters from $B$ : it says that $\left(b b_{1} b_{2}\right)=(b b b)=b$ for all $b, b_{1}, b_{2} \in B$; and it says that $\left(b b_{1} c\right)=(b b c)=g_{c}(b)$ and $\left(b c b_{1}\right)=(b c b)=h_{c}(b)$ for all $b, b_{1} \in B$, $c \in C$.

Our main result is a characterization of those collections of mappings $F$ that determine an SI differential mode. 
Lemma 3.4. Let $\mathbf{A}=\mathbf{B} \propto_{F} \mathbf{C}$ be a proper differential mode and consider the unary algebra $\mathbf{B}=(B, F)$. Then the congruence lattice of $\mathbf{B}$ is isomorphic to the interval $[0, \lambda]$ in the congruence lattice of $\mathbf{A}$, or briefly,

$$
\operatorname{Con}(\mathbf{B}) \simeq[0, \lambda]_{\mathbf{C o n}(\mathbf{A})}
$$

Proof. We prove that

(1) if $\beta$ is a congruence of $\mathbf{B}$, then $\beta \cup i d$ is a congruence of $\mathbf{A}$;

(2) if $\alpha \leq \lambda$ is a congruence of $\mathbf{A}$, then $\alpha \cap B^{2}$ is a congruence of $\mathbf{B}$.

Put $\varphi(\beta)=\beta \cup i d$ and $\psi(\alpha)=\alpha \cap B^{2}$. Both mappings preserve the order, $\psi \varphi(\beta)=$ $\beta$, and $\varphi \psi(\alpha)=\alpha$ whenever $\alpha \leq \lambda=B^{2} \cup i d$. Hence the two lattices are isomorphic.

To prove (1), put $\alpha=\beta \cup i d$. We need to check that $x \alpha u, y \alpha v, z \alpha w$ implies $(x y z) \alpha(u v w)$. If $x \in C$, then $(x y z)=x=u=(u v w)$. Now assume $x \in B$. If $y, z \in C$, then $y=v, z=w$ and $(x y z)=f_{y z}(x)=f_{v w}(x) \alpha f_{v w}(u)=(u v w)$. If $y \in C$ and $z \in B$, then $y=v$ and $(x y z)=(x y x)=h_{y}(x)=h_{v}(x) \alpha h_{v}(u)=$ $(u v u)=(u v w)$ using Proposition 2.1(2). The case $y \in B, z \in C$ is similar. And if $y, z \in B$, Proposition 2.1(1) gives $(x y z)=x \alpha u=(u v w)$.

The case (2) is rather obvious: let $\beta=\alpha \cap B^{2}$ and assume $x \beta y$. Then $f_{c d}(x)=$ $(x c d) \alpha(y c d)=f_{c d}(y), g_{c}(x)=(x x c) \alpha(y y d)=g_{c}(y)$, and $h_{c}(x)=(x c x) \alpha(y c y)=$ $h_{c}(y)$.

Theorem 3.5. A ternary algebra $\mathbf{A}$ is a proper SI differential mode if and only if $\mathbf{A}=\mathbf{B} \propto_{F} \mathbf{C}$ for some admissible collection of mappings $F$ such that the unary algebra $\mathbf{B}=(B, F)$ satisfies the following conditions:

(SI1) $\mathbf{B}$ is commutative and $|B|>1$.

(SI2) B is subdirectly irreducible.

(SI3) B does not contain disjoint subalgebras.

(SI4) (i) B contains no one-element subalgebra, or

(ii) for every $c$ at least one of the following takes place:

$f_{c c} \neq i d, g_{c} \neq i d, h_{c} \neq i d, f_{c d} \neq g_{d}$ for some $d, f_{d c} \neq h_{d}$ for some $d$.

(SI5) For every $c \neq d$, at least one of the following takes place:

$g_{c} \neq g_{d}, h_{c} \neq h_{d},\left|\left\{f_{c c}, f_{d d}, f_{c d}, f_{d c}\right\}\right|>1, f_{c e} \neq f_{d e}$ for some $e, f_{e c} \neq f_{\text {ed }}$ for some $e$.

Proof. $(\Rightarrow)$ Consider the representation $\mathbf{A}=\mathbf{B} \propto_{F} \mathbf{C}$ defined in Proposition 3.3. The mappings mutually commute by Lemma 3.1. The unary algebra $\mathbf{B}$ is SI according to Lemma 3.4. A subalgebra of $\mathbf{B}$ generated by an element $a \in B$ is exactly the orbit $\mathbf{R}_{\mathbf{A}}(a)$. Any two orbits intersect, because $\lambda=B^{2} \cup i d$. If condition (SI4) were to fail, we would have an element $b \in B$ fixed by all the mappings of $F$, and an element $c \in C$ that fails (ii). Then it is easy to check that $\{b, c\}^{2} \cup i d$ is a congruence that intersects $\lambda$ trivially. If condition (SI5) failed for certain elements $c, d \in C$, then it is easy to check that $\{c, d\}^{2} \cup i d$ is a congruence that intersects $\lambda$ trivially.

$(\Leftarrow)$ Assume the conditions are met and consider the algebra $\mathbf{A}=\mathbf{B} \propto_{F} \mathbf{C}$. Condition (SI1) ensures we obtained a differential mode (Lemma 3.1). Condition (SI3) says that $\lambda=B^{2} \cup i d$. (See the explanation in the previous part of the proof.) According to (SI2) and Lemma 3.4, in the interval $[0, \lambda]_{\mathbf{C o n}(\mathbf{A})}$, the intersection of non-trivial congruences is non-trivial. To prove that $\mathbf{A}$ is SI, it is sufficient to show that for every non-trivial congruence $\alpha$ of $\mathbf{A}$, the intersection $\alpha \cap \lambda$ is non-trivial. 
First, assume $b \alpha c$ for some $b \in B, c \in C$, and use (SI4). Either (i) holds and $\{b\}$ is not a subalgebra. Then there is a translation $R$ such that $R(b) \neq b$, and so $R(b) \alpha R(c)=c$, and we obtain a non-trivial pair $b(\alpha \cap \lambda) R(b)$. Or (ii) holds, and we have five cases.

- If $f_{c c}\left(b^{\prime}\right) \neq b^{\prime}$ for some $b^{\prime} \in B$, then $b^{\prime}=\left(b^{\prime} b b\right)(\alpha \cap \lambda)\left(b^{\prime} c c\right) \neq b^{\prime}$.

- If $g_{c}\left(b^{\prime}\right) \neq b^{\prime}$ for some $b^{\prime} \in B$, then $b^{\prime}=\left(b^{\prime} b b\right)(\alpha \cap \lambda)\left(b^{\prime} c b\right) \neq b^{\prime}$.

- If $h_{c}\left(b^{\prime}\right) \neq b^{\prime}$ for some $b^{\prime} \in B$, then $b^{\prime}=\left(b^{\prime} b b\right)(\alpha \cap \lambda)\left(b^{\prime} b c\right) \neq b^{\prime}$.

- If $f_{c d}\left(b^{\prime}\right) \neq g_{d}\left(b^{\prime}\right)$ for some $b^{\prime}$, then $f_{c d}\left(b^{\prime}\right)=\left(b^{\prime} b b\right)(\alpha \cap \lambda)\left(b^{\prime} c b\right)=g_{d}\left(b^{\prime}\right)$.

- If $f_{c d}\left(b^{\prime}\right) \neq h_{d}\left(b^{\prime}\right)$ for some $b^{\prime}$, then $f_{c d}\left(b^{\prime}\right)=\left(b^{\prime} b b\right)(\alpha \cap \lambda)\left(b^{\prime} b c\right)=h_{d}\left(b^{\prime}\right)$.

Second, assume $c \alpha d$ for some $c, d \in C, c \neq d$, and use (SI5).

- If $g_{c}(b) \neq g_{d}(b)$ for some $b \in B$, then $(b c b)(\alpha \cap \lambda)(b d b)$ is a non-trivial pair. Similarly for $h_{c}(b) \neq h_{d}(b)$.

- If $f_{u v}(b) \neq f_{u^{\prime} v^{\prime}}(b)$ for some $b \in B$ and $u, v, u^{\prime}, v^{\prime} \in\{c, d\}$, then (buv) $(\alpha \cap$ $\lambda)\left(b u^{\prime} v^{\prime}\right)$ is a non-trivial pair.

- If $f_{c e}(b) \neq f_{d e}(b)$ for some $b \in B$ and $e \in C$, then $(b c e)(\alpha \cap \lambda)$ (bde) is a non-trivial pair. Similarly for $f_{e c}(b) \neq f_{e d}(b)$.

In both cases, $\alpha \cap \lambda$ is non-trivial.

Conditions (SI4) and (SI5) may appear very technical, but they formalize the intuition that $F$ should not contain "redundant mappings", in the following sense. (SI4) says that if there is a common fixpoint $b \in B$ for all mappings from $F$ (hence $\{b\}$ is a one-element subalgebra), then no action caused by $c \in C$ should be trivial (or we could factor $b \sim c$ ). (SI5) says that the action by two different elements of $C$ should differ (or we could factor out similarly acting pairs).

Subdirectly irreducible commutative unary algebras were classified by Z. Ésik and B. Imreh in [1]. A unary algebra will be called cocyclic, if it is term equivalent to a $G$-set with $G=\mathbb{Z}_{p^{k}}$ for some prime $p$ and $k \in \mathbb{N} \cup\{\infty\}$. (Note that these are exactly the subdirectly irreducible abelian groups.) Now, subdirectly irreducible commutative unary algebras come in three families, and each member of the families is subdirectly irreducible:

(I) cocyclic unary algebras;

(II) disjoint union of a cocyclic unary algebra and a singleton unary algebra;

(III) unary algebras where the intersection of all subalgebras consists of a single element, satisfying a handful of combinatorial properties, to be described below.

To describe item (III), consider a commutative unary algebra $\mathbf{B}=(B, F)$ such that the intersection of all subalgebras consists of a single element $z$. Define a quasiorder relation $\leq$ by $a \leq b$ iff $b=t(a)$ for a term $t$. Hence $a \leq b$ precisely if $b$ is in the subalgebra generated by $a$. Let $a \equiv b$ iff $a \leq b$ and $b \leq a$. The relation $\equiv$ is indeed a congruence of $\mathbf{B}$. Now, according to [1], $\mathbf{B}$ is subdirectly irreducible if and only if the following conditions are met.

(N1) The intersection $Z$ of all at least two-element subalgebras of $\mathbf{B}$ is non-empty.

(N2) For every basic operation $f$ of $\mathbf{B}$, either $f(a) \equiv a$ for all $a$ and $f$ is a permutation, or $f(a) \not \equiv a$ for all $a \neq z$.

(N3) The unary algebra $(B \backslash\{z\}, G)$, where $G$ consists of all restrictions of mappings from $F$ that are permutations, is a disjoint union of pairwise isomorphic cocyclic algebras or singletons. 
(N4) For every $a \in B \backslash Z$ and every $b \neq a$, there is a term $t$ that represents a non-permutational mapping on $B$ such that

(i) $t(a) \neq t(b)$,

(ii) at least one of $t(a), t(b)$ belongs to the union of blocks from $M$, where $M$ is defined as the smallest subset of $\mathbf{B} / \equiv$ such that its complement has no maximal elements (in the quasiorder $\leq$ ).

Note that if $\mathbf{B}$ is finite, then $M=B / \equiv$, hence (ii) is trivially satisfied.

We shall call type (III) algebras nilpotent. (In the infinite case, this is not exactly correct terminology. Ésik and Imreh called such algebras separable quasi-nilpotent. We decided to stick to the shorter name, for we are mostly considering finite algebras.)

All type (II) algebras fail condition (SI3) of Theorem 3.5. The other two types give rise to SI differential modes, to be called cocyclic and nilpotent, respectively. Condition (SI4)(i) is satisfied by the cocyclic ones and fails for the nilpotent ones. We can restate our main theorem in the following way:

Corollary 3.6. A ternary algebra $\mathbf{A}$ is a proper SI differential mode if and only if $\mathbf{A}=\mathbf{B} \propto_{F} \mathbf{C}$ for some admissible collection of mappings $F$ such that the following conditions are satisfied:

(SI1-4) For the unary algebra $(B, F)$, one of the two options applies:

(I) it is cocyclic;

(III) it is nilpotent, and for every $c \in C$ at least one of the following holds:

$f_{c c} \neq i d, g_{c} \neq i d, h_{c} \neq i d, f_{c d} \neq g_{d}$ for some $d, f_{d c} \neq h_{d}$ for some $d$.

(SI5) For every $c \neq d$, at least one of the following holds:

$g_{c} \neq g_{d}, h_{c} \neq h_{d},\left|\left\{f_{c c}, f_{d d}, f_{c d}, f_{d c}\right\}\right|>1, f_{c e} \neq f_{d e}$ for some $e, f_{e c} \neq f_{\text {ed }}$ for some $e$.

We finish the section with a note on terminology. When we refer to the quasiorder of a nilpotent SI mode $\mathbf{A}=\mathbf{B} \propto_{F} \mathbf{C}$, we mean the quasiorder $\leq$ of the corresponding unary algebra $(B, F)$. Similarly for the congruence $\equiv$. The length of a nilpotent algebra, or a nilpotent SI differential mode, is defined to be the largest $n$ such that there is a chain $z>a_{1}>a_{2}>\ldots>a_{n}$, or infinity, if there are arbitrarily long finite chains. We say that the congruence $\equiv$ is trivial, if it is the identity relation, and hence $\leq$ is an order.

\section{The Szendrei CASE}

Many examples of modes, such as convex sets with barycentric operations, admit a sort of a linear representation, and thus satisfy a more restrictive variant of the entropic law, presented in [16] by Á. Szendrei. We use the name Szendrei identities, and the modes satisfying them are called Szendrei modes. For a ternary operation, the identities can be expressed as

$$
\left(\left(x_{11} x_{12} x_{13}\right)\left(x_{21} x_{22} x_{23}\right)\left(x_{31} x_{32} x_{33}\right)\right)=\left(\left(x_{\overline{11}} x_{\overline{12}} x_{\overline{13}}\right)\left(x_{\overline{21}} x_{2 \overline{2}} x_{\overline{23}}\right)\left(x_{\overline{31}} x_{\overline{32}} x_{\overline{33}}\right)\right)
$$

where ${ }^{-}$is a mapping that flips a single pair of indices $i j, j i$ and leaves the other pairs untouched. Hence, in the ternary case, there are exactly three different Szendrei identities. If $(\mathrm{R})$ holds, one of them is trivial and the other two say that

$$
((x y z) u v)=((x u z) y v) \quad \text { and } \quad((x y z) u v)=((x y v) u z) .
$$


It is easy to check that the two identities are equivalent (in modes) to a single one,

$$
(x y z)=((x y x) x z) .
$$

The importance of Szendrei identities is given by the following theorem [14][15]: A mode satisfies Szendrei identities if and only if it embeds into a semimodule over a commutative semiring. The theory of Szendrei differential modes is significantly simpler than the general one, see [7], and resembles that of differential groupoids.

Lemma 4.1. The algebra $\mathbf{B} \propto_{F} \mathbf{C}$ is a Szendrei mode if and only if the mappings from $F$ are mutually commutative and $f_{c d}=g_{d} h_{c}$ for every $c, d \in C$.

Proof. In one direction, (Sz) directly implies $f_{c d}(b)=(b c d)=((b c b) b d)=g_{d} h_{c}(b)$ for all $b \in B, c, d \in C$. In the other direction, the identity (Sz) is obviously satisfied if $x \in C$, so assume $x \in B$. If $y, z \in B$, use the fact that $B$ is a projection algebra. If $y \in B, z \in C$, then $(x y x)=x$, and so $((x y x) x z)=(x x z)=(x y z)$. For $y \in C$, $z \in B$ proceed similarly. And the last case, $y, z \in C$, describes the condition $f_{c d}=g_{d} h_{c}$.

Corollary 4.2. A ternary algebra $\mathbf{A}$ is a proper SI Szendrei differential mode if and only if $\mathbf{A}=\mathbf{B} \propto_{F} \mathbf{C}$ for some admissible collection of mappings $F$ such that the following conditions are satisfied:

(SI Sz) $f_{c d}=g_{d} h_{c}$ for every $c, d \in C$.

(SI1-4) For the unary algebra $(B, F)$, one of the two options applies:

(I) it is cocyclic;

(III) it is nilpotent, and for every $c \in C, g_{c} \neq i d$ or $h_{c} \neq i d$.

(SI5) For every $c \neq d$ in $C, g_{c} \neq g_{d}$ or $h_{c} \neq h_{d}$.

Condition (SI5) implies that the size of $C$ is bounded by the number of pairs of mappings $B \rightarrow B$, i.e.,

$$
|C| \leq\left(|B|^{|B|}\right)^{2}
$$

Actually, in the case of cocyclic SIs, the bound can be improved to $|B|^{2}$, since there are only $|B|$ mappings that preserve the cycle. There is no such bound in the general (non-Szendrei) case, as we shall see in the next section.

\section{Residual Bounds}

The residual bound of a variety is the smallest cardinal $\kappa$ such that all subdirectly irreducible algebras have size $<\kappa$. A variety is called residually large, if there is no such cardinal.

In the present section, we determine varieties of differential modes with finite residual bound. It turns out that there are no such non-Szendrei varieties (Theorem 5.4), and rather few Szendrei varieties (Theorem 5.5). Moreover, non-Szendrei varieties are residually large, and so are all the remaining locally finite Szendrei varieties.

The proof of Theorem 5.4 splits into two parts. First, we prove that every nonSzendrei variety contains a finite SI algebra of one of three special types. Then, we will discuss residual bounds for each of the three cases, reusing one of them in the Szendrei case.

Lemma 5.1. A variety containing a nilpotent SI differential mode $\mathbf{A}=\mathbf{B} \propto_{F} \mathbf{C}$ of length at least 2, also contains a nilpotent SI algebra $\mathbf{A}^{\prime}=\mathbf{B}^{\prime} \propto_{F^{\prime}} \mathbf{C}^{\prime}$ of length 2 such that the congruence $\equiv$ is trivial, the order $\leq$ is linear and $\left|C^{\prime}\right| \leq 2$. 
(The conditions imply that $\left|B^{\prime}\right|=3$ and $\left|A^{\prime}\right| \leq 5$.)

Proof. Define an equivalence $\alpha$ by $a \alpha b$ if and only if either $a, b \in B$ and $a \equiv b$, or $a=b \in C$. Clearly, $\alpha$ is a congruence of $\mathbf{A}$, and consider the algebra $\mathbf{A} / \alpha$. Choose $[a] \in B / \alpha$ in distance 2 from $[z]$ and a witness $\varphi \in F$ of this fact (i.e., $a \in B$ such that there is $b$ with $[a]<[b]<[z]$, and there is no $b^{\prime}$ with $[a]<\left[b^{\prime}\right]<[b]$ or $[b]<\left[b^{\prime}\right]<[z]$, and $\varphi:[a] \mapsto[b] \mapsto[z]$ in $\left.\mathbf{A} / \alpha\right)$. If it is possible to choose $\varphi=g_{c}$ or $\varphi=h_{c}$ or $\varphi=f_{c c}$ for some $c$, do so and let $C^{\prime}=\{c\}$. Otherwise, for $\varphi=f_{c d}$ put $C^{\prime}=\{c, d\}$. Let $\mathbf{A}^{\prime}$ be the subalgebra of $\mathbf{A} / \alpha$ generated by $\left\{[a],[c]: c \in C^{\prime}\right\}$. The corresponding unary algebra is certainly SI, nilpotent of length 2 , with trivial $\equiv$ and a linear order. If $\left|C^{\prime}\right|=1$, conditions (SI4) and (SI5) are trivial. If $\left|C^{\prime}\right|=2$, they follow from the fact that no single parameter translation is equal to $\varphi$. Hence, $\mathbf{A}^{\prime}$ is SI.

Lemma 5.2. Every non-Szendrei variety of differential modes contains a finite SI algebra $\mathbf{A}^{\prime}=\mathbf{B}^{\prime} \propto_{F^{\prime}} \mathbf{C}^{\prime}$ satisfying one of the following conditions:

(10) It is non-Szendrei cocyclic and $\left|C^{\prime}\right|=\{c, d\}$ with $f_{c d} \neq g_{d} h_{c}$.

(11) It is non-Szendrei nilpotent of length 1 and $\left|C^{\prime}\right|=\{c, d\}$ with $f_{c d} \neq g_{d} h_{c}$.

(12) It is nilpotent of length $2, \equiv$ is trivial, $\leq$ is linear and $\left|C^{\prime}\right| \leq 2$.

(In (10) and (11), it may happen that $c=d$.)

Proof. Every non-Szendrei variety of differential modes contains a non-Szendrei SI algebra $\mathbf{A}=\mathbf{B} \propto_{F} \mathbf{C}$. If it is nilpotent of length at least 2, we use Lemma 5.1. So assume it is either cocyclic, or nilpotent of length 1.

According to Lemma 4.1, there are $c, d \in C$ and $a \in B$ such that $f_{c d}(a) \neq$ $g_{d}\left(h_{c}(a)\right)$. If it is possible to choose $c=d$, do so; otherwise, pick arbitrarily. Let $\mathbf{A}^{\prime}$ be the subalgebra of $\mathbf{A}$ generated by $a, c, d$. It is certainly non-Szendrei. Since $a$ is not fixed by at least one of the mappings $f_{c d}, g_{d}, h_{c}$, it induces a non-trivial $\lambda$ block $B^{\prime}$. And there are at most two trivial $\lambda$ blocks, $\{c\}$ and $\{d\}$, hence $\mathbf{A}^{\prime}=\mathbf{B}^{\prime} \propto_{F^{\prime}} \mathbf{C}^{\prime}$. We check $\mathbf{A}^{\prime}$ satisfies conditions (SI4) and (SI5). If $c=d$, the conditions are trivial. Case $c \neq d$ : (SI4) since $f_{c d} \neq g_{d} h_{c}$, we get $h_{c} \neq i d$ or $f_{c d} \neq g_{d}$, and similarly also $g_{d} \neq i d$ or $f_{c d} \neq h_{c}$; (SI5) if we had $g_{c}=g_{d}$, then $f_{c c}=g_{c} h_{c}=g_{d} h_{c} \neq f_{c d}$.

Finitely generated subgroups of finite cyclic groups, or of the group $\mathbb{Z}_{p^{\infty}}$, are finite cyclic groups. Consequently, $\mathbf{A}^{\prime}$ will be finite even if $\mathbf{A}$ was not. If $\mathbf{A}$ was cocyclic, $\mathbf{A}^{\prime}$ will also be SI and cocyclic (possibly shorter) and meet condition (10). If $\mathbf{A}$ was nilpotent and $F^{\prime}$ contains only permutations, then $\mathbf{A}^{\prime}$ will again be SI and cocyclic and meet condition (10). Otherwise, $\mathbf{A}^{\prime}$ will meet condition (11).

A side remark: Lemma 5.1 does not assure that starting with a non-Szendrei algebra, we also obtain a non-Szendrei algebra. One can extend Lemma 5.2 in the following way: in the construction, if the initial algebra $\mathbf{A}$ is nilpotent of an arbitrary length, and if $\equiv$ is trivial, the resulting algebra $\mathbf{A}^{\prime}$ is also non-Szendrei nilpotent SI, with trivial $\equiv$ and linear order (not necessarily of length 2 ). However, if $\equiv$ is non-trivial on $\mathbf{A}$, then $\mathbf{A}^{\prime}$ may fail to be SI.

Lemma 5.3. A variety containing a finite SI differential mode satisfying condition (l0) or (l1) or (l2), is residually large.

Proof. Let $\mathbf{A}=\mathbf{B} \propto_{F} \mathbf{C}$ be such an SI differential mode. We provide three constructions for the respective types. 
(10) Denote $B=\left\{0, \ldots, p^{k}-1\right\}$, and assume the mapping $x \mapsto x+1 \bmod$ $p^{k}$ generates the right multiplication monoid (actually group) $\mathbf{R}_{\mathbf{A}}$ (we implicitly assume the mapping acts identically on $C$ ). Let $n$ be a cardinal number and put

$$
A^{\prime}=B^{(n)} \cup\left\{u \in A^{n} \text { : there is } i \text { such that } u_{i} \in C \text {, and } u_{j} \in B \text { for all } j \neq i\right\} \text {. }
$$

Here and later in the proof, $X^{(n)}$ denotes the set of all $x \in X^{n}$ such that all but finitely many entries are zero. Clearly $\mathbf{A}^{\prime}$ is a subalgebra of $\mathbf{A}^{n}$ and we define a congruence $\alpha$ on $\mathbf{A}^{\prime}$ such that $u \alpha v$ if and only if

- either $\sum u_{i} \equiv \sum v_{i}\left(\bmod p^{k}\right)$,

- or $u_{i}=v_{i} \in C$ for some $i$.

The factor $\mathbf{A}^{\prime} / \alpha$ has exactly $|B|+n \cdot|C|$ elements, to be denoted

- $[0], \ldots,\left[p^{k}-1\right]$, depending on the sum of the entries $\bmod p^{k}$,

- [ci], for the block of vectors with $c \in C$ at position $i$.

Then, for every $a, b, b^{\prime} \in B, c, d \in C, i, j<n$, and $u, v$ arbitrary,

$$
\begin{array}{ll}
([c i][u][v])=[c i], & \left([a][b]\left[b^{\prime}\right]\right)=[a], \\
([a][b][c i])=\left[g_{c}(a)\right], & ([a][c i][b])=\left[h_{c}(a)\right], \\
([a][c i][d j])=\left[g_{d} h_{c}(a)\right] \text { if } i \neq j, & ([a][c i][d i])=\left[f_{c d}(a)\right] .
\end{array}
$$

Hence the congruence $\lambda$ on $\mathbf{A}^{\prime} / \alpha$ has a single non-trivial block $\{[b]: b \in B\}$, the corresponding unary algebra is again cocyclic, and

$$
g_{[c i]}=g_{c}, \quad h_{[c i]}=h_{c}, \quad f_{[c i][d i]}=f_{c d}, \text { and } f_{[c i][d j]}=g_{d} h_{c} \text { if } i \neq j .
$$

We check that the algebra $\mathbf{A}^{\prime} / \alpha$ satisfies condition (SI5).

Case $|C|=1$. Denote $C=\{c\}$ and assume $f_{c c} \neq g_{c} h_{c}$. Then, for $[c i] \neq[c j]$, take $e=[c j]$ and note that $f_{[c i][c j]}=g_{c} h_{c} \neq f_{c c}=f_{[c j][c j]}$.

Case $|C|=2$. Denote $C=\{c, d\}$ in a way that $f_{c d} \neq g_{d} h_{c}$.

- For $[c i] \neq[d i]$, use (SI5) for $\mathbf{A}$.

- For $[c i] \neq[c j]$, take $e=[d j]$ and note that $f_{[c i][d j]}=g_{d} h_{c} \neq f_{c d}=f_{[c j][d j]}$.

- For $[d i] \neq[d j]$, take $e=[c i]$ and note that $f_{[c i][d j]}=g_{d} h_{c} \neq f_{c d}=f_{[c i][d i]}$.

- For $[c i] \neq[d j], i \neq j$, either $g_{c} \neq g_{d}$ and thus $g_{[c i]} \neq g_{[d j]}$; or $f_{c d} \neq g_{c} h_{c}$ and thus $f_{[c j][d j]} \neq f_{[c j][c i]}$; or both $g_{c}=g_{d}$ and $f_{c d}=g_{c} h_{c}$, but in such case $g_{c} h_{c}=f_{c d} \neq g_{d} h_{c}=g_{c} h_{c}$, contradiction.

(11) Denote $B=\left\{z, 0, \ldots, p^{k}-1\right\}$, where $z$ is the terminal point and $0, \ldots, p^{k}-1$ are the elements of the other $\equiv$ block (possibly $k=0$ ), and assume the mapping $x \mapsto x+1 \bmod p^{k}$ generates the right multiplication monoid $\mathbf{R}_{\mathbf{A}}$. The construction is very similar to the previous one. Let $n$ be a cardinal number and put

$$
A^{\prime}=B^{(n)} \cup\left\{u \in A^{n} \text { : there is } i \text { such that } u_{i} \in C \text {, and } u_{j} \in B \text { for all } j \neq i\right\} .
$$

Clearly $\mathbf{A}^{\prime}$ is a subalgebra of $\mathbf{A}^{n}$ and we define a congruence $\alpha$ on $\mathbf{A}^{\prime}$ such that $u \alpha v$ if and only if

- either $u, v \in(B \backslash\{z\})^{(n)}$ and $\sum u_{i} \equiv \sum v_{i}\left(\bmod p^{k}\right)$,

- or $u, v \in B^{(n)}$ and both contain the $z$ entry,

- or $u_{i}=v_{i} \in C$ for some $i$.

The factor $\mathbf{A}^{\prime} / \alpha$ has exactly $|B|+n \cdot|C|$ elements, to be denoted as above, $[0], \ldots,\left[p^{k}-1\right]$ and $[z]$ and $[c i]$, respectively. Formulas for the operation are exactly as in case (10), hence the congruence $\lambda$ on $\mathbf{A}^{\prime} / \alpha$ also has a single non-trivial block $\{[b]: b \in B\}$, and the corresponding unary algebra is SI nilpotent of length 1 . It 
satisfies condition (SI5) for the very same reason it did in the previous case, and condition (SI4) follows immediately from the fact that it was satisfied by $\mathbf{A}$.

(12) Denote $B=\{x, y, z\}$, and assume the mapping $x \mapsto y \mapsto z \mapsto z$, to be called the shift mapping, generates the right multiplication monoid $\mathbf{R}_{\mathbf{A}}$. By (N2), $\mathbf{R}_{\mathbf{A}}$ actually consists of the identity mapping, the shift mapping and the constant mapping onto $z$.

If there is $c \in C$ such that one of $g_{c}, h_{c}, f_{c c}$ is the shift mapping, replace $C$ by $\{c\}$ and obtain a new SI differential mode satisfying (12), to be used for the construction. Let $n \geq 2$ be a cardinal number and put

$A^{\prime}=\left(B^{n} \backslash\{x\}^{n}\right) \cup\left\{u \in A^{n}\right.$ : there is $i$ such that $u_{i} \in C$, and $u_{j} \in B$ for all $\left.j \neq i\right\}$.

Clearly $\mathbf{A}^{\prime}$ is a subalgebra of $\mathbf{A}^{n}$ and we define a congruence $\alpha$ on $\mathbf{A}^{\prime}$ such that $u \alpha v$ if and only if

- either there is a single coordinate $i$ such that $u_{i}=v_{i}=y$, and $u_{j}=v_{j}=x$ for all $j \neq i$,

- or both $u, v$ have exactly two entries equal $y$ and the other entries equal $x$ (on possibly different coordinates),

- or $u, v \in B^{n}$ and do not meet any of the preceding conditions,

- or $u_{i}=v_{i} \in C$ for some $i$.

The factor $\mathbf{A}^{\prime} / \alpha$ has exactly $n+2+n \cdot|C|$ elements, to be denoted

- $[i], i<n$, for the block of vectors with single $y$ at position $i$,

- $[y]$, for the block of vectors with precisely two $y$ 's,

- $[z]$, for the block of remaining vectors from $B^{n}$,

- and $[c i]$ for the block of vectors with $c \in C$ at position $i$.

It is easy to see that the congruence $\lambda$ on $\mathbf{A}^{\prime} / \alpha$ has again a single non-trivial block. The congruence $\equiv$ is trivial (hence (N3) holds), and its elements are ordered $[i]>[y]>[z]$ for every $i<n$ (hence (N1) holds). To check (N2), consider a translation $\varphi$ in $\mathbf{A}^{\prime} / \alpha$ fixing some $[i]$ or fixing $[y]$. Note that $\varphi$ acts non-trivially in at most two coordinates, and the involved translations in $\mathbf{A}$ have to fix $x$ or $y$ at these positions. Hence, by (N2) in $\mathbf{A}$, both are identities and thus $\varphi$ is identity too. For (N4), to separate $[i],[j]$ with $i \neq j$, consider the translation acting as the shift mapping in the $i$-th coordinate and identically in the other coordinates. Consequently, the corresponding unary algebra is SI nilpotent of length 2 (now with a non-linear order).

We check $\mathbf{A}^{\prime} / \alpha$ satisfies conditions (SI4) and (SI5). Let $\varphi, \psi \in R_{\mathbf{A}}$ and $\varphi^{\prime}, \psi^{\prime}$ be the mappings on $\mathbf{A}^{\prime} / \alpha$ acting as $\varphi, \psi$, respectively, in the $i$-th coordinate, and identically in the other coordinates. Observe that if $\varphi \neq \psi$, then $\varphi^{\prime}([j]) \neq \psi^{\prime}([j])$ for every $j \neq i$. Now, (SI4) follows immediately from the observation. So does (SI5) for all pairs $[c i] \neq[d i]$ for every $c, d \in C$. For the remaining pairs, we consider two cases.

Case $|C|=1$. Denote $C=\{c\}$. One of $g_{c}, h_{c}, f_{c c}$ is the shift mapping. Assume it is $g_{c}$ (the other options are proved similarly). Then $g_{[c i]}([i])=[z] \neq[y]=g_{[c j]}([i])$, hence $g_{[c i]} \neq g_{[c j]}$ for every $i \neq j$, and (SI5) holds.

Case $|C|=2$. Denote the elements $c, d$ in a way that $f_{c d}$ is the shift mapping. Then, for every $i \neq j, f_{[c i][d i]}([j])=[y]$, but $f_{[u i][v j]}([j]) \neq[y]$ for every choice of $u, v \in C$. Hence, for every $[c i] \neq[c j], f_{[c i][d i]} \neq f_{[c j][d i]}$; for every $[d i] \neq[d j]$, $f_{[c i][d i]} \neq f_{[c i][d j]}$; and for every $[c i] \neq[d j], f_{[c i][d i]} \neq f_{[d j][d i]}$. So (SI5) holds for all pairs. 
Theorem 5.4. Every non-Szendrei variety of differential modes is residually large.

Proof. Immediate consequence of Lemmas 5.2 and 5.3.

Now we turn to the Szendrei case. Contrary to the general case, we have a bound on the size of an SI algebra in terms of the size of its non-trivial $\lambda$ block. Consequently, some varieties turn out to be residually rather small.

In [7] we proved that every subvariety of Szendrei differential modes is relatively based by identities in two variables, and that the non-trivial subvarieties are in 1-1 correspondence with congruences of the monoid $\left(\mathbb{N}_{0},+\right)^{2}$ in the following way: to a congruence $\zeta$, assign the subvariety $\mathcal{V}_{\zeta}$ relatively based by

$$
(((((x \underbrace{x y) \ldots) x y}_{i}) \underbrace{y x) \ldots) y x}_{j})=(((((x \underbrace{x y) \ldots) x y}_{k}) \underbrace{y x) \ldots) y x}_{l})
$$

for every $(i, j) \zeta(k, l)$. This defines an order reversing bijection between the two lattices. The least congruence corresponds to the variety of Szendrei differential modes, the largest congruence to the variety of projection algebras. We will refer to varieties by the corresponding congruences.

Theorem 5.5. Let $\mathcal{V}_{\zeta}$ be a variety of Szendrei differential modes given by a congruence $\zeta$.

(1) $\mathcal{V}_{\zeta}$ has a finite residual bound if and only if there are $m, n>1$ such that $(m, 0) \zeta(1,0)$ and $(0, n) \zeta(0,1)$

(2) If $\mathcal{V}_{\zeta}$ is locally finite and the previous condition fails, it is residually large.

Proof. An SI Szendrei differential mode $\mathbf{A}=\mathbf{B} \propto_{F} \mathbf{C}$ belongs to $\mathcal{V}_{\zeta}$ if and only if $g_{c}^{i} h_{c}^{j}=g_{c}^{k} h_{c}^{l}$ for every $c \in C$ and every $(i, j) \zeta(k, l)$. Given a mapping $f$, the smallest $r$ and $s$ such that $f^{r}=f^{r+s}$ will be called the length and the period of $f$ (if they exist). Note that in a locally finite variety, there is a bound on the length and period of every translation in every algebra.

$(\Leftarrow)$ The identities force that all right translations have bounded period $p \leq$ $\operatorname{lcm}(m-1, n-1)$ and length at most 1 . Consequently, all SIs in $\mathcal{V}_{\zeta}$ are either nilpotent of length 1 , or cocyclic. In the nilpotent case, $|B| \leq p+1$, in the cocyclic case, $|B| \leq p$. According to the notes following Corollary 4.2 , any SI in $\mathcal{V}_{\zeta}$ has size at most $(p+1)+(p+1)^{2(p+1)}$.

$(\Rightarrow)$ Assume the condition fails. It means the equation

$$
(((x \underbrace{y z) \ldots) y z}_{k})=(x y z)
$$

fails in $\mathcal{V}_{\zeta}$ for every $k$. This must be witnessed by subdirectly irreducible algebras in $\mathcal{V}_{\zeta}$. If there is a nilpotent SI of length at least 2 in $\mathcal{V}_{\zeta}$, we can use Lemmas 5.1 and 5.3 and are done. If there is no such, i.e., if all SIs in $\mathcal{V}_{\zeta}$ are cocyclic or nilpotent of length 1 , then $\mathcal{V}_{\zeta}$ is not locally finite, and either there is an SI with an infinite $\lambda$ block (the $\mathbb{Z}_{p^{\infty}}$ action), or there are (infinitely many) SIs with arbitrarily large finite $\lambda$ blocks. In either case, the residual bound of $\mathcal{V}_{\zeta}$ is not finite.

\section{Related PRoblems}

Theorem 3.5 and the Ésik-Imreh classification of subdirectly irreducible commutative unary algebras bring an insight into the rather intricate structure of subdirectly irreducible algebras in an interesting class of strongly solvable modes. We 
wish to point out a connection of our results to two major open problems of universal algebra that concern subdirectly irreducible algebras.

One of the motivations behind our work was, to solve Park's problem for modes. It asks whether varieties with a finite signature and finite residual bound are finitely based (for a background information, see [17]). Park conjectured an affirmative answer. It follows from our results that Park's conjecture holds for all varieties of differential modes. Actually, for a rather trivial reason: Theorem 5.4 says that all varieties of differential modes with finite residual bound are Szendrei; but all varieties of Szendrei differential modes are finitely based, as proved in [7] by reduction to Rédei's finiteness theorem for commutative semigroups. (A non-Szendrei variety with no finite base was constructed in [5].)

The other problem I had in mind, the $R S$ problem, asks whether there is a finitely generated variety with a finite signature and residual bound $\aleph_{0}$ (for a background information, see [6]). According to our results, there is no such variety of differential modes.

Both questions have been answered assuming certain Mal'tsev conditions: for congruence modular varieties using commutator theory, and also for congruence meet-semidistributive varieties. Searching for a (counter)example, one shall focus on varieties satisfying no non-trivial Mal'tsev condition. Perhaps not as simple as differential modes, yet this is a part of my motivation to study strongly solvable modes.

Acknowledgement. I wish to thank Michał Stronkowski for his interest in my work, for reading a preliminary version of this text and suggesting several ideas that improved Section 5.

\section{REFERENCES}

[1] Z. Ésik, B. Imreh, Subdirectly irreducible commutative automata, Acta Cybernetica 5 (1981), $251-260$.

[2] K. Kearnes, The structure of finite modes, manuscript, 1990's.

[3] K. Kearnes, Semilattice modes I: the associated semiring, Algebra Universalis 34 (1995), 220272.

[4] K. Kearnes, Subdirectly irreducible modes, Discuss. Math. Algebra Stochastic Methods 19/1 (1999), 129-145.

[5] A. Kravchenko, A. Pilitowska, A. Romanowska, D. Stanovský, Differential modes, Internat. J. Algebra Comput. 18/3 (2008), 567-588.

[6] R. McKenzie, The residual bounds of finite algebras, Internat. J. Algebra Comput. 6/1 (1996), $1-28$.

[7] A. Pilitowska, A. Romanowska, D. Stanovský, Varieties of differential modes embeddable into semimodules, Internat. J. Algebra Comput. 19/5 (2009), 669-680.

[8] J. Płonka, On k-cyclic groupoids, Math. Jap. 30/3 (1985), 371-382.

[9] A. Romanowska, On some representations of groupoid modes satisfying the reduction law, Dem. Math. 21 (1988), 943-960.

[10] A. Romanowska and B. Roszkowska, On some groupoid modes, Dem. Math. 20 (1987), 277290.

[11] A. Romanowska and B. Roszkowska, Representations of $n$-cyclic groupoids, Algebra Universalis 26/1 (1989), 7-15.

[12] A. Romanowska, J. D. H. Smith, Differential groupoids, Contributions to General Algebra 7 (1991), 283-290.

[13] A. Romanowska, J. D. H. Smith, Modes, World Scientific, 2002.

[14] D. Stanovský, Idempotent subreducts of semimodules over commutative semirings, Rend. Semin. Mat. Univ. Padova 121 (2009), 33-43. 
[15] M. M. Stronkowski, Embedding entropic algebras into semimodules and modules, Internat. J. Algebra Comput. 19/8 (2009), 1025-1047.

[16] Á. Szendrei, Identities satisfied by convex linear forms, Algebra Universalis 12 (1981), 103122.

[17] R. Willard, The finite basis problem, Contributions to General Algebra 15 (2004), 199-206.

Charles University in Prague

KA MFF UK, Sokolovská 83, 18675 Praha, Czech Republic

E-mail address: stanovsk@karlin.mff.cuni.cz 